\title{
Aspectos sensoriais e cognitivos do controle postural
}

\author{
Sensorial and Cognitive Aspects of Postural Control
}

\section{Regiane Luz Carvalho' ${ }^{1}$, Gil Lúcio Almeida²}

\section{RESUMO}

A estabilização postural depende de mecanismos periféricos relativamente simples assim como de mecanismos complexos envolvendo altos níveis de função cognitiva e integração sensório-motora. A teoria de controle baseada em respostas reflexas hierarquicamente organizadas tem cedido lugar a uma visão sistêmica que enfatiza a múltipla organização e interação neural. È tema de discussão a forma de organização das informações sensoriais e do processamento cognitivo e sua relação com a elaboração das estratégias motoras. Buscando responder a estas perguntas este artigo revisa estudos que abordam aspectos biomecânicos, sensoriais e cognitivos do controle postural.

Unitermos. Equilíbrio Musculosquelético, Limiar Sensorial, Postura

Citação. Carvalho RL, Almeida GL. Aspectos sensoriais e cognitivos do controle postural. Rev Neurocienc 2008; in press.

Trabalho realizado na Universidade Estadual de Campinas/SP.

1.Fisioterapeuta, Doutora em Biologia Funcional/ Neurofisiologia-Unicamp, Professora Adjunto III do curso de Fisioterapia da PUC-Minas Campus Poços de Caldas.

2.Fisioterapeuta, Doutor Child Development -University of Illinois at Chicago, Pós-doutorado pelo Rush Medical Center e University of Illinois at Chicago, Professor e diretor dos programas da New York Institute of Technology (NYIT) no Brasil.

\section{SUMMARY}

The postural stabilization are subserved by multiple sensory and motor mechanisms, ranging from relatively simple, peripheral mechanisms to complex ones involving the highest levels of cognitive function and sensory-motor integration. The reflex/hierarchical concept of postural control has changed to a systems approach, which emphasizes a goal-directed neural organization of multiple, interacting systems. However, there is some discussion about how sensory information and cognitive processing are organized to control balance. To answer these questions, this paper reviews the biomechanics, sensorial and cognitive aspects of postural control.

Keywords. Musculoskeletal Equilibrium, Sensory Thresholds, Posture

Citation. Carvalho RL, Almeida GL. Sensorial and Cognitive Aspects of Postural Control. Rev Neurocienc 2008; in press. 


\section{INTRODUÇÃO}

$\mathrm{Na}$ última década os mecanismos envolvidos no controle postural têm sido amplamente investigados. A teoria de controle baseada em respostas reflexas hierarquicamente organizadas e desencadeadas por informações sensoriais independentes tem cedido lugar a uma visão sistêmica, que enfatiza a múltipla organização e interação neural. Esta nova visão sugere que o controle postural emerge da interação entre indivíduo, tarefa e ambiente, não podendo ser mais visto como simples resposta reativa a um estímulo sensorial, mas sim como uma habilidade baseada na experiência, intenção e adaptação ${ }^{1}$.

$\mathrm{O}$ controle postural eficiente é fundamental para o sucesso de grande parte das tarefas diárias. $\mathrm{O}$ conhecimento dos aspectos neurológicos e biomecânicos deste controle tem implicações diretas para a fisioterapia, justificando a importância de uma revisão que aborde a evolução deste conhecimento.

\section{MÉTODO}

Para este fim foram consultados os bancos de dados Medline, Lilacs e Web of Science, com as seguintes palavras-chave: postural control, sensorial system, visual, vestibular, somatosensorial e cognitive. Para seleção dos artigos inicialmente foi feita a leitura dos resumos verificando seu enquadramento nos seguintes critérios de inclusão: idioma português ou inglês, período de 1993-2008, revisões sistemáticas sobre controle postural e seus aspectos sensoriais e cognitivos e estudos experimentais relacionados ao efeito da manipulação das informações sensoriais e cognitivas no controle postural. Foram selecionados 5 artigos de revisão, 22 estudos experimentais e 2 capítulos de livro para leitura na íntegra e inclusão nesta revisão.

\section{RESULTADOS}

O controle motor engloba aspectos aplicados tanto ao controle do movimento quanto ao controle postural $^{2}$. O controle postural envolve a orientação postural e o equilíbrio. A orientação postural é definida como a habilidade de manter a relação apropriada entre os segmentos corporais e o ambiente. Depende do controle do alinhamento corporal e do tônus em relação à gravidade, à superfície de suporte, às referências internas e às informações sensoriais. Já o equilíbrio postural se refere à habilidade de manter a posição do corpo, especificamente do centro de massa, dentro dos limites de estabilidade através da inter-relação das várias forças que agem sobre o corpo, incluindo a força de gravidade, dos músculos e inerciais. Pode-se dizer que a tarefa básica do equilíbrio é a manutenção da estabilidade corporal tanto em condição estática quanto dinâmica ${ }^{3}$.

O controle postural é considerado uma habilidade motora complexa derivada da interação dos sistemas neural e musculoesquelético. Os componentes neurais envolvem o processamento motor, processamento sensorial, representação interna e altos níveis de processamento essenciais para os aspectos adaptativos e antecipatórios do controle postural ${ }^{4}$. Os altos níveis de processamento neural se referem às influências cognitivas no controle postural como atenção e motivação e não ao controle consciente propriamente dito.

Os componentes musculoesqueléticos incluem aspectos como a amplitude de movimento, flexibilidade, propriedades dos músculos e relações biomecânicas entre os segmentos. Os aspectos biomecânicos são caracterizados pelas propriedades visco-elásticas e pela configuração anatômica dos ossos, músculos e articulações. Qualquer limitação de força, amplitude de movimento, dor ou mesmo de controle dos pés (base de suporte) irá afetar o controle postural ${ }^{3}$.

As informações dos múltiplos sistemas sensoriais incluindo o somatossensorial, visual e vestibular são integradas pelo sistema de controle motor para orientar e alinhar a posição entre os segmentos corpóreos e a sua localização em relação ao meio externo $^{5}$. A partir destas informações o sistema nervoso elabora estratégias posturais que representam soluções sensório-motoras para o controle da postura incluindo não apenas sinergias musculares, mas também padrões de movimentos articulares, torques e forças de contato ${ }^{6,7}$.

A interação dos sistemas sensoriais não resulta de uma simples convergência, mas sim de transformações apropriadas e coordenadas. Cada canal sensorial tem qualidades diferentes em termos de resolução e importância, sendo que a fidedignidade de uma informação pode alterar a confiabilidade de outra $^{8}$. Quando o ambiente sensorial é alterado, ocorre a readequação da dominância das informações sensoriais para minimizar os conflitos ${ }^{9}$. Vários estudos têm investigado o efeito da manipulação sensorial e sua relação com o controle postural.

\section{Visão}

A função básica da visão é orientar a posição e movimento da cabeça em relação ao ambiente, sendo que em condições estáticas as aferências visuais 
reduzem a oscilação corporal em aproximadamente $50 \%{ }^{10}$. Para Vallis et al. ${ }^{11}$, além de sua função básica, a visão desempenha um papel fundamental na estabilização tardia das correções posturais e no planejamento de reações antecipatórias.

O papel das informações visuais na estabilização postural parece aumentar durante a permanência em superfícies instáveis, durante a aquisição de uma habilidade motora e em pacientes com desordens vestibulares ${ }^{12,13}$.

As informações visuais podem ser facilmente manipuladas pela movimentação do cenário, oclusão ou por estímulos optocinéticos ${ }^{14,15}$.

\section{Informações somatossensoriais}

O sistema somatosensorial difere de outros sistemas por apresentar receptores pelo corpo e não concentrados em locais especializados e por responder a vários estímulos que se agrupam em quatro categorias: toque, temperatura, dor e propriocepção ${ }^{9}$. Os receptores proprioceptivos têm especial relação com o controle postural por informarem continuamente ao SNC a posição de cada segmento corpóreo em relação a outro possibilitando a representação da geometria estática e dinâmica do corpo ${ }^{9}$. A importância da propriocepção para a manutenção da postura fica evidente pelas conseqüências clínicas desastrosas observadas em indivíduos com déficits nesta modalidade sensorial ${ }^{16}$.

Existem evidências do predomínio destas informações na manutenção do equilíbrio em superfícies estáveis ${ }^{17}$. Nesta condição, o risco de queda é vinte e três vezes maior em portadores de neuropatia periférica ${ }^{18}$.

A propriocepção pode ser manipulada por várias técnicas como bloqueio isquêmico dos músculos, anestesia local e vibração dos tendões musculares. A utilização limitada da isquemia e anestesia se deve ao efeito não seletivo e de curta duração. A vibração muscular altera a orientação postural por ativar as aferências fusais produzindo uma situação de ilusão da posição do membro. Como resultado da ativação fusal observa-se a contração muscular ${ }^{19}$.

\section{Informações vestibulares}

O sistema vestibular envia informações sobre a orientação da cabeça detectando as variações temporais das velocidades angular e linear'. O papel das informações vestibulares no controle das respostas posturais tem sido muito debatido. Day et al..$^{20}$ defendem a premissa de que as informações vestibulares desempenham um pequeno papel na manutenção de postu- ras estáticas. Por outro lado, a redução das aferências vestibulares levam a déficits consideráveis no controle de tarefas complexas como a locomoção e o balanço em plataforma sinusoidal, indicando sua grande importância em situações de natureza dinâmica ${ }^{21}$.

As informações vestibulares podem ser manipuladas pela aplicação de estimulação galvânica (EGV). A aplicação de corrente galvânica nos processos mastóideos despolariza o nervo vestibular e aumenta a freqüência de disparo dos aferentes vestibulares do lado do cátodo e diminui do lado do ânodo, resultando em uma oscilação corporal para o lado do ânodo ${ }^{21}$. A EGV permite avaliar os efeitos primários das informações vestibulares na postura já que outras técnicas, como a rotação cefálica, ativam simultaneamente as aferências proprioceptivas cervicais ${ }^{22}$.

A mesma estimulação vestibular resulta em respostas posturais diferentes dependendo do nível de ativação dos sistemas somatossensorial e visual, indicando a existência de uma ampla integração intersensorial ${ }^{5,17}$. Por exemplo, a oscilação decorrente da EGV é menor quando as informações visuais estão disponíveis e maior durante a permanência em uma superfície de espuma ou plataforma de translação ${ }^{23}$.

\section{Processamento cognitivo}

A relação entre atenção e controle postural é uma área nova e crescente de estudo que tem revelado aspectos importantes do papel do processamento cognitivo no controle postural. O método mais utilizado para este fim é o paradigma de duas tarefas, no qual o controle postural (considerado como tarefa primária) e uma tarefa secundária são realizados concomitantemente ${ }^{4}$.

Ebersbach et al..$^{24}$ analisaram o efeito de tarefas concorrentes [retenção de memória (repetir seqüência de números) e execução de movimento fino (abrir e fechar botão)] no controle postural durante o movimento (marcha) e observaram uma piora durante a execução da dupla tarefa. Lajoie et al. ${ }^{25}$ compararam o tempo de resposta a um estímulo sonoro em diversas posições: sentado, em pé e sobre uma barra estreita. Descobriram que quanto maior a dificuldade de manutenção da postura requerida, maior era o tempo de resposta ao estímulo sonoro. Estes achados indicam que a instabilidade postural aumenta o tempo necessário para realização de uma tarefa cognitiva e prejudica a sua performance. Para Woollacott et al. ${ }^{4}$ a necessidade de atenção para o controle postural depende da complexidade da tarefa. 
Avançando para clínica, Horak ${ }^{3}$ sugere que a ocupação com uma segunda tarefa pode resultar em processamento cognitivo insuficiente, levando a quedas em pacientes com distúrbios neurológicos.

\section{Estratégias Posturais}

Estudos de controle motor levaram ao conceito de sinergias musculares e estratégias posturais. As sinergias podem ser definidas como padrões de atividade muscular em resposta a perturbações, organizadas centralmente de forma a simplificar o controle do movimento. As estratégias posturais incluem não apenas as sinergias musculares, mas também padrões de movimento, torques articulares e forças de contato. As estratégias posturais também são descritas como respostas posturais automáticas coordenadas $^{26}$.

São três as principais estratégias utilizadas para retornar o corpo à posição de equilíbrio ${ }^{3}$.

Estratégiadotornozelo,naqualocorposemove ao nível do tornozelo como um pêndulo invertido. É utilizada para manutenção do equilíbrio frente a pequenas oscilações ${ }^{3}$.

Estratégia do quadril, que é utilizada quando a base de suporte se torna menor e mais instável. Os movimentos que fazem parte da estratégia do quadril são centrados nesta articulação e se caracterizam pela ativação precoce da musculatura proximal do tronco e quadril ${ }^{3}$.

Estratégia do passo, que é utilizada em grandes perturbações para evitar a queda, sendo caracterizada pela ativação inicial dos abdutores do quadril e co-contração do tornozelo ${ }^{3}$.

Para Horak et al. ${ }^{1}$, as estratégias emergem do processamento neural para fornecer um plano de ação baseado nos objetivos, no contexto ambiental, e particularmente na atividade ou tarefa. São organizadas no espaço e no tempo para produzir forças efetivas que contraponham o distúrbio. Indivíduos sem comprometimento neurológico modulam a magnitude de sua resposta postural automática com a magnitude do distúrbio. Com a exposição repetida a distúrbios inesperados às estratégias posturais, que inicialmente são executadas com ativação muscular excessiva, apresentam uma redução na magnitude de sua resposta ${ }^{27}$.

O conceito de estratégia postural também pode ser aplicado às respostas posturais antecipatórias. A aquisição destas implica na transformação de correções posturais compensatórias em antecipatórias. $\mathrm{O}$ modelo geral para esta transformação envolve um sis- tema adaptativo que constrói uma imagem interna do distúrbio a ser minimizado. Os ajustes posturais antecipatórios dependem da experiência prévia ${ }^{26}$.

Indivíduos com pobre coordenação das respostas posturais automáticas demonstram maior instabilidade em resposta a distúrbios externos. Já indivíduos com pobre coordenação das reações antecipatórias desequilibram mais com seus próprios movimentos ${ }^{1}$.

\section{Implicações práticas}

O conhecimento da organização sensorial e cognitiva e de aspectos específicos das estratégias posturais pode ajudar na compreensão do controle postural e de suas disfunções. A avaliação do controle postural deve permitir a quantificação da capacidade funcional do paciente, assim como das alterações específicas que comprometem seu controle postural. Deve abranger as características sensoriais, especificidades das estratégias posturais, assim como fatores relacionados à atenção. Medidas simples do equilíbrio podem não identificar as limitações do processo sensório-motor responsáveis pelo descontrole postural, como, por exemplo, os problemas na organização temporal das estratégias posturais observados em vários pacientes com distúrbios neurológicos ${ }^{1}$. Dentre estes problemas destacam-se a lentidão no início da resposta postural e a seqüência temporal reversa de ativação dos músculos posturais ${ }^{28}$.

Outras limitações específicas podem estar relacionadas à dificuldade de adaptação das estratégias posturais às alterações ambientais, ou mesmo às restrições músculo-esqueléticas como fraqueza e inabilidade de gerar força suficiente para contrapor um distúrbio postural ${ }^{3}$. O processamento sensorial também tem grande influência do controle postural e, quando inadequado, pode prejudicar o desenvolvimento de modelos internos precisos do controle postural, comprometer a capacidade de adaptação e prejudicar a habilidade de antecipação ${ }^{26}$.

A identificação de déficits posturais específicos pode auxiliar a reabilitação, aumentando as chances de sucesso. A latência das respostas posturais pode ser trabalhada com estimulações sensoriais que aumentem a excitabilidade dos motoneurônios e facilitem seu disparo ${ }^{26}$. A ordem de recrutamento muscular pode ser trabalhada com feedback ${ }^{29}$. A capacidade de adaptação aos distúrbios pode ser estimulada pela exposição repetida a perturbações de várias amplitudes, assim como pela variação da precisão das informações sensoriais ${ }^{26}$. 


\section{CONSIDERAÇÕES FINAIS}

O controle da postura depende de uma complexa interação entre o sistema neural e músculo-esquelético. $\mathrm{O}$ sistema nervoso utiliza as informações sensoriais na elaboração de comandos motores capazes de recuperar o equilíbrio. Estes comandos são modulados pelas características sensoriais e também por mecanismos relacionados à atenção, experiência e contexto. $\mathrm{O}$ sucesso do controle postural depende da flexibilidade e adaptação da organização sensorial e das estratégias de manutenção do equilíbrio. O fisioterapeuta deve acompanhar os avanços do conhecimento nesta área e estar atento a todos os fatores capazes de influenciar o controle postural, pois só assim será capaz de direcionar adequadamente a intervenção terapêutica.

\section{REFERÊNCIAS}

1. Horak FB, Henry SM, Shumway-Cook A. Postural perturbations: new insights for treatment of balance disorders. Phys Ther 1997;77(Suppl 5):517-33.

2. Massion J. Postural systems in developmental perspective. Neurosci Biobehav Rev 1998;22(4):465-72.

3. Horak FB. Postural orientation and equilibrium: what do we need to know about neural central of balance to prevent falls. Age and Ageing 2006;35:117-21.

4. Woollacott M, Shumway-Cook. Attention and the control of posture and gait: a review of an emerging area of research. Gait and Posture 2002;16:1-14.

5. Bacsi AM, Colebatch JG. Evidence for reflex and perceptual vestibular contributions to postural control. Exp Brain Res 2005;160(Suppl) $1: 22-8$.

6. Krishnamoorthy V, Latash ML, Scholz, JP, Zatsiorsky VM. Muscle synergies during shifts of the center of pressure by standing persons. Exp Brain Res 2003;152:281-92.

7. Ting LH. Dimensional reduction in sensorimotor systems: a framework for understanding muscle coordination of posture. Prog Brain Res 2007;165:299-321.

8. Lackner JR, DiZio P. Vestibular, proprioceptive, and haptic contributions to spatial orientation. Annu Rev Psychol 2005;56:115-47.
9. Mochizuki L, Amadio AC. As informações sensoriais para o controle postural. Fisioter Mov 2006;19:11-8.

10. Sasaki O, Usami S, Gagey P, Martinerie J, Quyen M, Arranz P. Role of visual input in nonlinear postural control system. Exp Brain Res 2002;147:1-7

11. Vallis LA, Patla AE, Adkin AL. Control of steering in the presence of unexpected head yaw movements. Exp Brain Res 2001;138:128-34.

12. Redfern MS, Yardly L, Bronstein PA. Visual influences on balance. J Anxiety Disord 2001;15:81-94.

13. Pozzo T, Levik Y, Berthoz A. Head and trunk movements in the frontal plane during complex dynamic equilibrium tasks in humans. Exp Brain Res 1995;106(Suppl 2):327-38.

14. Kuo AD, Speers RA, Peterka RJ, Horak FB. Effect of altered sensory conditions on multivariate descriptors of human postural sway. Exp Brain Res 1998;122(Suppl 2):185-95.

15. Tanahashi S, Ujike H, Kozawa R, Ukai K. Effects of visually simulated roll motion on vection and postural stabilization. J Neuroeng Rehabil 2007;9(4):39.

16. Nardone A, Grasso M, Schieppati M. Balance control in peripheral neuropathy: are patients equally unstable under static and dynamic conditions? Gait Posture 2006;23(3):364-16.

17. Mergner T, Huber W, Becker W. Vestibular Neck interaction and transformation of sensory coordinates. J Vest Res 1997;7:347-67.

18. Richardson JK, Hurvitz EA. Peripheral neuropathy: a true risk factor for falls. J Am Geriatr Soc 1992;40:1008-12.

19. Michel-Pellegrino V, Amoud H, Hewson DJ, Duchene J. Identification of a degradation in postural equilibrium invoked by different vibration frequencies on the tibialis anterior tendon. Conf Proc IEEE Eng Med Biol Soc 2006;1:4047-50.

20. Day BL, Cauquil A, Bartolomei L, Pastor MA, Lyon IN. Human body segment tilts induced by galvanic stimulation: a vestibularly driven balance protection mechanism. J Physiol 1997;500:601-72.

21. Peruch P, Borel L, Gaunet F, Thinus-Blanc G, Magnan J, Lacour M. Spatial performance of unilateral vestibular defective patients in nonvisual versus visual navigation. J Vestib Res 1999;9(Suppl 1):37-47.

22. Fitzpatrick RC, Day BL. Probing the human vestibular system with galvanic stimulation. J Appl Physiol 2004;96:2301-16.

23. Horak FB, Hlavacka F. Somatosensory loss increases vestibulospinal sensitivity. J Neurophysiol 2001;86:575-85.

24. Ebersbach G, Dimitrijevic MR, Poewe W. Influence of concurrent tasks on gait: a dual task approach. Percept Mot Skills 1995;81:107-13.

25. Lajoie Y, Teasdale N, Bard C, Fleury M. Attentional demands for static and dynamic equilibrium. Exp Brain Res 1993;97:139-44.

26. Shummway-Cook A, Woollacott M. Motor Control: theory and practical applications. Baltimore: Williams \&Wilkins, 1995, pp. 119-41.

27. Horak FB. Adaptation of automatic postural responses. In: Bloedel J, Wise SP. Acquisition of Motor Behavior in Vertebrates. Catabridge: MIT Press, 1996, pp. 57-85.

28. Aruin AS, Almeida GL. A. coactivation strategy in anticipatory postural adjustments in person with Down Syndrome. Motor Control 1997;1:178-91

29. Di Fabio RP, Badke MB, MaEoy A, Ogden E. Kinematic properties of voluntary sway in patients with unilateral primary hemispheric lesions. Exp Brain 1990;5(Suppl 13):248-5. 